This Section of Epidemiology and Psychiatric Sciences regularly appears in each issue of the Journal to describe relevant studies investigating the relationship between neurobiology and psychosocial psychiatry in major psychoses. The aim of these Editorials is to provide a better understanding of the neural basis of psychopathology and clinical features of these disorders, in order to raise new perspectives in every-day clinical practice.

Paolo Brambilla, Section Editor and Michele Tansella, Editors EPS

\title{
Language disturbances in ADHD
}

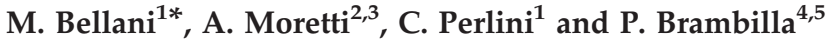 \\ ${ }^{1}$ Section of Psychiatry and Clinical Psychology, Department of Public Health and Community Medicine, Inter-University Center for Behavioural \\ Neurosciences (ICBN), University of Verona, Verona, Italy \\ ${ }^{2}$ IRCCS 'E. Medea' Scientific Institute, San Vito al Tagliamento, Italy \\ ${ }^{3}$ University of Udine, Udine, Italy \\ ${ }^{4}$ Department of Experimental Clinical Medicine, Inter-University Center for Behavioural Neurosciences (ICBN), University of Udine, Udine, Italy \\ ${ }^{5}$ IRCCS 'E. Medea' Scientific Institute, Udine, Italy
}

\begin{abstract}
This article aims to review the studies exploring language abilities in attention deficit hyperactivity disorder (ADHD; with or without comorbid language impairment) focusing on oral speech discrimination, listening comprehension, verbal and spatial working memory as well as on discourse analysis and pragmatic aspects of communication and language comprehension.
\end{abstract}

Received 11 June 2011; Revised 13 June 2011; Accepted 18 June 2011

Key words: ADHD, language impairment, pragmatics, working memory.

According to the DSM-IV-TR (APA, 2000), the Attention Deficit Hyperactivity Disorder (ADHD) is characterized by persistent and severe levels of hyperactivity/impulsivity and/or symptoms of inattention, interfering with normal functioning in social, educational and working environments. Even if not included in the core diagnostic criteria of ADHD, language disturbances may often be present (Baker \& Cantwell, 1992; Camarata \& Gibson, 1999), affecting both linguistic and pragmatic domains. Indeed, hyperactive/impulsive symptoms may result in speaking without thinking or respecting the conversational turn in conversations, interrupting others' speech and talking excessively. These symptoms may reflect an association between ADHD and difficulties in pragmatic aspects of communication. Interestingly, such

* Address for correspondence: Dr M. Bellani, Department of Public Health and Community Medicine, Section of Psychiatry and Clinical Psychology, University of Verona, Piazzale L.A. Scuro 10, 37134 Verona, Italy.

Fax: +39,-045-8027498 (Email: marcella.bellani@univr.it; paolo. brambilla@uniud.it) kinds of impairments (i.e. inappropriate and impulsive behaviours in conversations and relationships), which have been reported in ADHD (Oram et al. 1999; Kim \& Kaiser, 2000) (Table 1), are somewhat similar to those described in pervasive developmental disorders (Bishop \& Baird, 2001; Geurts \& Embrechts, 2008) (Table 1) and schizophrenia (Tavano et al. 2008; Bellani et al. 2009, 2010).

Inattentive symptoms appear to be linked also with language comprehension difficulties, since children do not apparently listen and do not follow teacher's instructions. In their study, Baker \& Cantwell (1992) realized indeed that there is a strong association between language impairments (LI) and ADHD (Table 1). Achievement and cognition problems are related to both conditions, and so it is a challenge to define which deficits belong to ADHD, which ones to LI alone and which ones are shared by the two conditions, although the presence of LI is suggested to represent the crucial factor (Cohen et al. 2000).

Other authors have mainly focused on working memory abilities in ADHD children, with or without language impairments, reporting different results 
Table 1. Studies exploring the association between language disturbances and ADHD

\begin{tabular}{|c|c|c|c|c|}
\hline Study & Subjects & $\begin{array}{l}\text { Children age } \\
\text { range (years) }\end{array}$ & Language tests & Findings \\
\hline $\begin{array}{l}\text { Baker \& Cantwell } \\
\quad(1992)\end{array}$ & $\begin{array}{l}\text { Children with: ADHD + SL } \\
\text { disorder }(n=65)\end{array}$ & $6.0-15.3$ & $\begin{array}{l}\text { - Goldman-Fristoe Test of Articulation } \\
\text { - Denver Articulation Screening Test } \\
\text { - PPVT } \\
\text { - Receptive-Expressive-Emerging Language Scale } \\
\text { - Test of Auditory Comprehension of Language } \\
\text { - Token Test for Children } \\
\text { - Illinois Test of Psycholinguistic Abilities } \\
\text { - Carrow Elicited Language Inventory } \\
\text { - Memory for Sentences Test } \\
\text { - Detroit Test of Learning Aptitude }\end{array}$ & $\begin{array}{l}\text { All children presented linguistic deficits: 78\% speech } \\
\text { articulation, } 69 \% \text { language-processing, 58\% expressive } \\
\text { language, 34\% receptive language. }\end{array}$ \\
\hline Oram et al. (1999) & $\begin{array}{l}\text { Children with: ADHD }(n=25) \\
\text { ADHD + LI }(n=28) \\
\text { non-ADHD controls }(n=24)\end{array}$ & $7-11$ & $\begin{array}{l}\text { - Test of Word Finding } \\
\text { - Rosner's Auditory Analysis Test } \\
\text { - CELF-R }\end{array}$ & $\begin{array}{l}\text { ADHD-only children had poor performance on the CELF-R } \\
\text { Formulated Sentences subtest. }\end{array}$ \\
\hline Cohen et al. (2000) & $\begin{array}{l}\text { Children with: ADHD + LI }(n= \\
\text { 36), ADHD }(n=69), \mathrm{OPD}+ \\
\text { LI }(n=30), \text { OPD }(n=31)\end{array}$ & $7-14$ & $\begin{array}{l}\text { - } \text { PPVT-R } \\
\text { - } \text { EOWPVT-R } \\
\text { - TROG } \\
\text { - } \text { TOLD Grammatic Understanding and Grammatic } \\
\text { Comprehension } \\
\text { - CELF-R } \\
\text { - } \text { DTLA-3-Word Sequences and Story Construction } \\
\text { - TAAS } \\
\text { - } \text { PAT } \\
\text { - The Pragmatics Checklist }\end{array}$ & $\begin{array}{l}\text { ADHD + LI and OPD + LI children had poorer language, } \\
\text { pragmatic and narrative skills than ADHD and OPD } \\
\text { children (exceptions: CELF-R Word Structures subtest } \\
\text { and PAT). }\end{array}$ \\
\hline Kim \& Kaiser (2000) & $\begin{array}{l}\text { Children with: ADHD }(n=11) \text {, } \\
\text { TD children }(n=11)\end{array}$ & $6-8$ & $\begin{array}{l}\text { - } \text { PPVT-R } \\
\text { - TOLD-2 Primary } \\
\text { - TOPL } \\
\text { - the Pragmatic Protocol }\end{array}$ & $\begin{array}{l}\text { In ADHD children poorer performances on the TOLD-2 } \\
\text { sentence imitation and word articulation subtests, } \\
\text { speaking quotient and speech-language quotient. More } \\
\text { inadequate pragmatic behaviours. }\end{array}$ \\
\hline
\end{tabular}

PPVT

nois Test of Psycholinguistic Abilities

guage Inventory

Test of Word Finding

- Rosner's Auditory Analysis Test

TROG

TOLD Gramma

- CELF-R

- DILA-3-Word Sequences and Story Construction

TAAS

- the Pragmatic Protocol 




Jonsdottir et al. (2005)

Martinussen \&

Tannock (2006)

Mathers (2006)

Parents $(P)$ and Teachers $(T)$ of $\quad 5-17$

$$
\text { 19), Normal Controls }(n=19)
$$$$
\begin{aligned}
& \text { ADHD + LI }(n=18), \text { LI }(n= \\
& \text { 19), Normal Controls }(n=19)
\end{aligned}
$$

Children with: ADHD-C + SLI $\quad 81 \frac{12-121 / 2}{2}$ $(n=19)$, ADHD-C non-SLI $(n=15)$, Normal Controls $(n$ $=15$ )

Children with: ADHD $(n=62), \quad 7-13$ $\mathrm{ADHD}+\mathrm{RD} / \mathrm{LI}(n=32), \mathrm{RD} /$

LI $(n=15)$, Normal Controls $(n=34)$

Children with: ADHD $(n=11), \quad 8-12$ TD children $(n=11)$

- CCC

ADHD group had low scores on the CCC pragmatic composite similar to Asperger or PDDNOS groups, as evaluated by Parents and Teachers.

- PPVT-III

- EVT

- CELF-3 Receptive Language and Expressive

Language

- Word Attack WRMT-R

- Block Design WISC-III

- Narrative and Expository Passage Comprehension Tasks

- Comprehension Monitoring Ability-Error

Detection Tasks

- Verbal and Spatial Memory tasks (Span and

Working Memory measures)

- $\mathrm{K}-\mathrm{ABC}$

- TOLD-2I

- Digits Forward and Digits Backward subtests from the WISC-III

- Finger Windows task from the WRAML

- Use of an interactive software to generate an animated cartoon

- Three language-sampling tasks (providing a story retell text, providing a recount text and providing a procedural text)

- Writing texts task
The ADHD patients comprehension was impaired in listening to spoken expository passages (inferences and monitoring of instructions), poorer verbal working memory, spatial span and spatial working memory.

ADHD-C + SLI children had poorer performance only on verbal working memory tasks.

Worse verbal storage in children with RD/LI and ADHD + $\mathrm{RD} / \mathrm{LI}$. Deficits in visual-spatial storage and verbal and visual-spatial central executive functions in all clinical groups.

In ADHD children more abandoned utterances in spoken texts, spelling and punctuation errors in written texts and more tangential and unconnected information. 


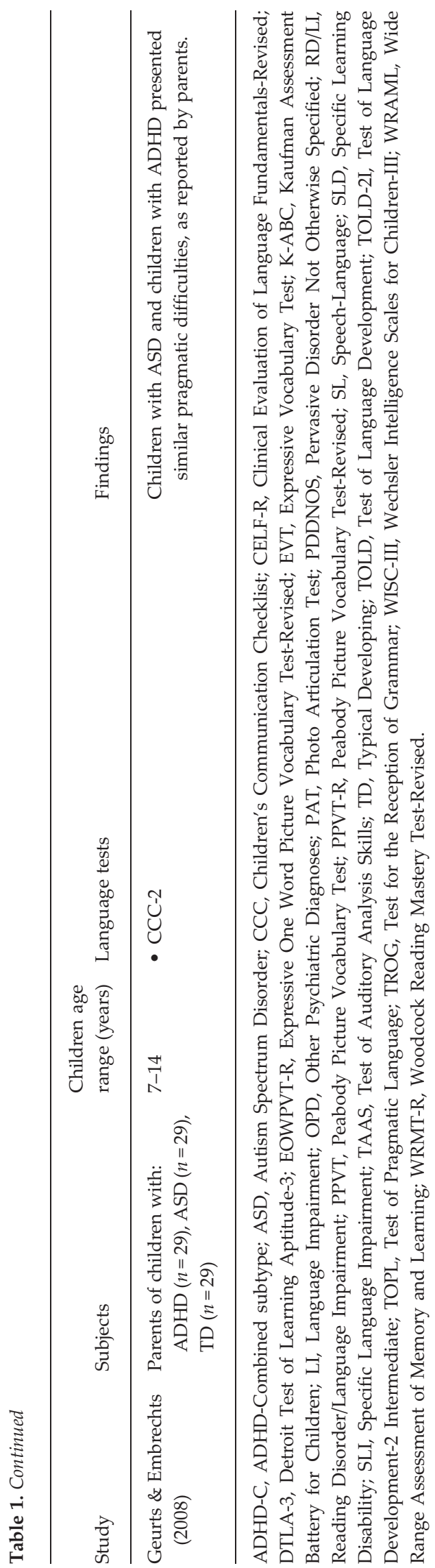

(McInnes et al. 2003; Jonsdottir et al. 2005; Martinussen \& Tannock, 2006) (Table 1). For example, McInnes et al. (2003) described altered listening comprehension, spatial span, and verbal and spatial working memory in ADHD children without comorbid LI. In contrast, Jonsdottir et al. (2005) showed that working memory abilities were impaired only in ADHD children with language problems. Martinussen \& Tannock (2006) noted that working memory may be compromised independently of comorbid reading or language deficits in ADHD. Additionally, in 2006, Mathers analysed the texts of ADHD children observing more abandoned utterances, spelling and punctuation errors, avoidance, tangential and unconnected information in comparison with typical developing children (Table 1).

In conclusion, pragmatic aspects, verbal working memory and discourse analysis seem to be affected in ADHD, being related to language abilities but, partially, also to general executive functions (Cohen et al. 2000). Therefore, comorbidity with language disorders in children with ADHD should consistently be detected and, when present, taken into account for intervention strategies, being a good indicator of inattention. Future studies should further characterize the correlations between language impairments and higher cognitive dimensions, trying to plan innovative and specific interventions for ADHD with or without LI.

\section{References}

APA, American Psychiatric Association (2000). Diagnostic and Statistical Manual of Mental Disorders, DSM-IV-TR, 4th ed. Text Revised, APA, Washington, DC.

Baker L, Cantwell D (1992). Attention deficit disorder and speech/language disorders. Comprehensive Mental Health Care 2, 3-16.

Bellani M, Perlini C, Brambilla P (2009). Language disturbances in schizophrenia. Epidemiologia e Psichiatria Sociale 18, 314-317.

Bellani M, Dusi N, Brambilla P (2010). Longitudinal imaging studies in schizophrenia: the relationship between brain morphology and outcome measures. Epidemiologia e Psichiatria Sociale 19, 207-10.

Bishop DVM, Baird G (2001). Parent and teacher report of pragmatic aspects of communication: use of the Children's Communication Checklist in a clinical setting. Developmental Medicine and Child Neurology 43, 809-818.

Camarata S, Gibson T (1999). Pragmatic language deficits in attention-deficit hyperactivity disorder. Mental Retardation and Developmental Disabilities Research Reviews 5, 207-214.

Cohen NJ, Vallance DD, Barwick M, Im N, Menna R, Horodezky NB, Isaacson L (2000). The interface between ADHD and language impairment: an examination of language, achievement, and cognitive processing. Journal of 
Child Psychology and Psychiatry and Allied Disciplines 41, 353-362.

Geurts HM, Embrechts M (2008). Language profiles in ASD, SLI, and ADHD. Journal of Autism and Developmental Disorders 38, 1931-1943.

Jonsdottir S, Bouma A, Sergeant JA, Scherder EJA (2005).

The impact of specific language impairment on working memory in children with ADHD combined subtype. Archives of Clinical Neuropsychology 20, 443-456.

Kim OH, Kaiser AP (2000). Language characteristics of children with ADHD. Communication Disorders Quarterly 21, 154-165.

Martinussen R, Tannock R (2006). Working memory impairments in children with attention-deficit hyperactivity disorder with and without comorbid language learning disorder. Journal of Clinical and Experimental Neuropsychology 28, 1073-1094
Mathers ME (2006). Aspects of language in children with ADHD. Applying functional analyses to explore language use. Journal of Attention Disorders 9, 523-533.

McInnes A, Humphries T, Hogg-Johnson S, Tannock R (2003). Listening comprehension and working memory are impaired in attention-deficit hyperactivity disorder irrespective of language impairment. Journal of Abnormal Child Psychology 31, 427-443.

Oram J, Fine J, Okamoto C, Tannock R (1999). Assessing the language of children with attention deficit hyperactivity disorder. American Journal of Speech-Language Pathology 8, $72-80$.

Tavano A, Sponda S, Fabbro F, Perlini C, Rambaldelli G, Ferro A, Cerruti S, Tansella M, Brambilla P (2008). Specific linguistic and pragmatic deficits in Italian patients with schizophrenia. Schizophrenia Research 102, $53-62$. 